Revised version

Original article: Physica A 394 (2014) 166-176

DOI: http://dx.doi.org/10.1016/j.physa.2013.09.054

\title{
A complex network analysis of hypertension-related genes
}

\author{
Huan Wang ${ }^{\mathrm{a}}$, Chuan-Yun $\mathrm{Xu}^{\mathrm{a}}$, Jing-Bo Hu${ }^{\mathrm{b}}, \mathrm{Ke}-\mathrm{Fei} \mathrm{Cao}^{\mathrm{a}, *}$ \\ ${ }^{a}$ Center for Nonlinear Complex Systems, Department of Physics, School of Physics Science and Technology, Yunnan University, \\ Kunming, Yunnan 650091, China \\ ${ }^{b}$ Department of Electronic and Electrical Engineering, Baoji University of Arts and Sciences, Baoji, Shaanxi 721016, China
}

\begin{abstract}
In this paper, a network of hypertension-related genes is constructed by analyzing the correlations of gene expression data among the Dahl salt-sensitive rat and two consomic rat strains. The numerical calculations show that this sparse and assortative network has small-world and scale-free properties. Further, 16 key hub genes (Col4a1, Lcn2, $C d k 4$, etc.) are determined by introducing an integrated centrality and have been confirmed by biological/medical research to play important roles in hypertension.
\end{abstract}

Keywords: Complex network; Hypertension; Integrated centrality; Hub gene

\section{Introduction}

Since the fundamental work on random graphs by Erdős and Rényi [1], especially the seminal works on the small-world phenomenon by Watts and Strogatz [2] and the scale-free property by Barabási and Albert [3], the study of complex networks has received extensive attention. Scientists have found that most real networks are neither completely regular nor completely random, but usually display a small-world effect and/or a scale-free behavior [4, 5, 6, 7]. The research results show that complex network theory has been proven to be a powerful tool for the analysis in various fields, such as the World Wide Web [8, 9], social networks [10], ecological systems [11], traffic systems [12, 13], and so on.

In biological systems, elements that interact or regulate each other can be represented by a network, namely, a collection of nodes and edges (links) [14, 15, 16, 17, 18, 19]. At the simplest level, the individual elements are described as nodes and their interactions are reduced to edges connecting between pairs of nodes. Here, the networkbased approach is an effective way to discover the collective action of the individual parts, namely, the systems-level behavior [16, 20, 21, 22], which has been widely used in biochemical and medical research and offers a conceptual framework to understand molecular mechanisms and disease pathologies.

Hypertension is a major risk factor for cardiovascular morbidity and mortality; the worldwide prevalence of hypertension is $25 \%$ [23, 24]. Over the years, a great deal of research shows that the arterial blood pressure of many essential hypertensive patients exhibits an increased sensitivity to dietary salt intake, which is known as the saltsensitive (SS) hypertension [25, 26]. These people account for about 50\% of hypertensive patients [27]. Therefore,

\footnotetext{
${ }^{*}$ Corresponding author. Tel.: +86 87165031605 .

Email addresses: whh227@126.com (Huan Wang), kmchyxu@gmail.com (Chuan-Yun Xu), joyboble@126.com (Jing-Bo Hu), kf cao163@163.com (Ke-Fei Cao)
} 
the studies about SS hypertension contribute to a decrease in the level of blood pressure and in the incidence of mortality due to cardiovascular complications, and thus improve human health [28, 29]. Existing research for the Dahl SS rat [30, 31] and consomic rat strains [32, 33] reveals that salt and genetic factors have a major impact on hypertension [34, 35, 36]. During the last 30 years or so, the clinical research and treatment of hypertension have improved dramatically [37, 38, 39]. However, its molecular mechanisms and pathologies involved remain intricate and elusive.

In the present work, we attempt to study the genes that are involved in SS hypertension using the complex network approach. We will propose a simple rule to construct the network model of hypertension-related genes, where the nodes are individual genes and the connections are derived from the expression correlations that are based on microarray data. Through calculating several statistical indices and analyzing topological characteristics of the network, we find out key hub genes that play significant roles in hypertension and describe their functions. Based on both biological knowledge and network theory, this study confirms the role of key genes in hypertension from another perspective and provides an idea for studying the relation between genes associated with hypertension.

This paper is organized as follows. In Section 2 we introduce the construction of the network model of hypertensionrelated genes. In Section 3, we analyze the statistical and topological characteristics of the gene network, and determine key hub genes by introducing an integrated centrality. The biological descriptions of hub genes are presented in Section 4 , while Section 5 gives concluding remarks.

\section{Construction of network model of hypertension-related genes}

The Dahl SS rat is a widely used genetic model of human hypertension, which was proposed by Dahl et al. in the early 1960s [30, 31]. It develops hypertension upon exposure to a high-salt intake. The consomic rat strains, used as the normotensive control for the Dahl SS rat, are generated by substituting a chromosome or a part of a chromosome from a normal rat strain for the corresponding genomic region of the SS rat [32, 33, 36]. Previous research showed that substitution of chromosome 13 or 18 could significantly attenuate hypertension [34, 35]. The two consomic rat strains (generated by substituting chromosomes 13 and 18, respectively) have genetic homology with the Dahl SS rat, but lead to the amelioration of hypertension; and the segregation of hypertensive phenotype is regarded as the result of different gene expression patterns among the three rat strains caused by substituting chromosomes. Therefore, our study focuses on the analysis of gene expression data (GED) among the Dahl SS rat and two consomic rat strains: $H$ (GED for SS rat with high blood pressure), $S^{\mathrm{I}}$ (GED for substitution of chromosome 13) and $S^{\mathrm{II}}$ (GED for substitution of chromosome 18), where all three strains of rats were maintained on a high sodium intake for two weeks and gene expression profiles were examined using microarrays (see http://pga.mcw.edu). All data of $H, S^{\mathrm{I}}$ and $S^{\mathrm{II}}$ are given in three columns (SS2wk, SS13 2wk, and SS18 2wk) in Table S2 of Supplemental Figures and Tables of Ref. [36]. In Table 1 we list detailed data of some randomly selected genes and feature genes calculated in Section 3 .

Let us consider the network $G_{H}=\left(V_{H}, E_{H}\right)$, where $V_{H}=\left\{v_{i}\right\}(i=1,2, \ldots, N)$ is the set of $N$ nodes, and $E_{H}=\left\{v_{i}, v_{j}\right\}$ the set of edges or connections between nodes. We will use the following notation: $A_{i j}=1$ indicates that there is an edge between nodes $v_{i}$ and $v_{j}$; and $A_{i j}=0$ otherwise. Our gene network model is constructed from the correlations based on GED in three rat strains in two steps, which is designed to explore the relationship between genetic change trend and hypertensive phenotype.

Step 1. Calculation of change ratios of GED: The $N=335$ hypertension-related genes are served as nodes of the network, each node represents an individual gene shown as gene symbol or CloneID (for the gene without a gene symbol). For each of the 335 nodes, we define the change ratios $R_{i}^{\mathrm{I}}$ and $R_{i}^{\mathrm{II}}$ of GED between $\left\{S_{i}^{\mathrm{I}}, S_{i}^{\mathrm{II}}\right\}$ and $H_{i}$ as follows:

$$
\left\{\begin{array}{l}
R_{i}^{\mathrm{I}}=\left(S_{i}^{\mathrm{I}}-H_{i}\right) /\left|H_{i}\right|, \\
R_{i}^{\mathrm{II}}=\left(S_{i}^{\mathrm{II}}-H_{i}\right) /\left|H_{i}\right|,
\end{array}\right.
$$

where $i=1,2, \ldots, 335$. Then we can calculate the change ratios $R_{i}^{\mathrm{I}}$ and $R_{i}^{\mathrm{II}}$ separately (these data are shown as $R^{\mathrm{I}}$ and $R^{\mathrm{II}}$ in Table 1). It is easy to obtain the averages of two groups of change ratios $\left\{R_{i}^{\mathrm{I}}\right\}$ and $\left\{R_{i}^{\mathrm{II}}\right\}$, respectively: $T^{\mathrm{I}}=0.150725$, and $T^{\mathrm{II}}=0.141180$; which can be used as thresholds to determine whether a connection should be made between two nodes in the next step. It should be indicated that there are many ways of choosing the threshold, a higher threshold would result in a higher average degree and a greater influence or disturbance among nodes (genes), whereas a lower threshold would result in fewer connections but a greater possibility of the actual correlation of genes. So the average change ratio would be a reasonable choice of the threshold to make reliable connections and a 
Table 1: Some GED $\left(H, S^{\mathrm{I}}\right.$ and $\left.S^{\mathrm{II}}\right)$ of three rat strains and change ratios $\left(R^{\mathrm{I}}\right.$ and $\left.R^{\mathrm{II}}\right)$ of two groups. In this paper, we use "Hist $1 h 2 a \imath^{\prime \prime}$ as the abbreviation of gene symbol "Hist1h2ai_predicted /// Hist1h4a_predicted".

\begin{tabular}{|c|c|c|c|c|c|}
\hline Gene & $H$ & $S^{1}$ & $S^{11}$ & $R^{1}$ & $R^{\text {II }}$ \\
\hline Ssg1 & 0.760115 & 0.039832 & 0.084195 & -0.947598 & -0.889234 \\
\hline Lcn2 & 0.915950 & 0.089252 & 0.114858 & -0.902558 & -0.874602 \\
\hline Ociadl & -0.410164 & 0.075833 & 0.108042 & 1.184886 & 1.263413 \\
\hline $\mathrm{Nrld} 2$ & 0.503251 & 0.036939 & 0.056712 & -0.926599 & -0.887308 \\
\hline Tagln & 0.765658 & 0.130215 & 0.223917 & -0.829931 & -0.707550 \\
\hline$F z d 2$ & 0.474655 & 0.086283 & 0.068861 & -0.818220 & -0.854925 \\
\hline RGICL83 & 0.456126 & -0.024038 & 0.072557 & -1.052701 & -0.840928 \\
\hline$C d c 2 a$ & 0.583242 & 0.106746 & 0.118771 & -0.816978 & -0.796360 \\
\hline Colec12 & 0.450878 & 0.117010 & 0.230425 & -0.740483 & -0.488942 \\
\hline RGIEJ34 & 0.628989 & 0.075356 & 0.140478 & -0.880196 & -0.776660 \\
\hline RGIHD68 & 0.608212 & 0.213125 & 0.258199 & -0.649589 & -0.575479 \\
\hline Mcm6 & 0.255819 & 0.112129 & 0.061829 & -0.561684 & -0.758311 \\
\hline Ctsd & 0.606232 & 0.088551 & 0.151729 & -0.853932 & -0.749719 \\
\hline Casp6 & 0.501480 & 0.101879 & 0.095765 & -0.796843 & -0.809036 \\
\hline Timp1 & 0.589334 & 0.103506 & 0.122895 & -0.824368 & -0.791467 \\
\hline Ctsl & 0.277923 & -0.046910 & 0.023243 & -1.168789 & -0.916368 \\
\hline MCWA09_96 & 0.372041 & 0.049722 & 0.087101 & -0.866352 & -0.765883 \\
\hline Nudt4 & -0.368880 & -0.142909 & -0.061423 & 0.612586 & 0.833487 \\
\hline Histlh2ai & 0.532325 & 0.100425 & 0.099606 & -0.811346 & -0.812884 \\
\hline MCWA13_68 & 0.432117 & 0.049337 & 0.096514 & -0.885824 & -0.776649 \\
\hline Hfe & 0.339892 & 0.069570 & 0.055555 & -0.795316 & -0.836552 \\
\hline MCWA10_48 & 0.488626 & 0.061358 & 0.087510 & -0.874427 & -0.820907 \\
\hline Rbpl & 0.487553 & 0.019423 & 0.108073 & -0.960162 & -0.778336 \\
\hline$C d k 4$ & 0.297970 & 0.043814 & 0.051296 & -0.852959 & -0.827847 \\
\hline$S d c 1$ & 0.447238 & 0.047824 & 0.105806 & -0.893068 & -0.763424 \\
\hline Fbnl & 0.290349 & 0.047677 & 0.075306 & -0.835793 & -0.740637 \\
\hline Gpnmb & 0.374620 & -0.004214 & 0.052804 & -1.011249 & -0.859047 \\
\hline MCWA11_14 & 0.260709 & 0.063897 & 0.033333 & -0.754912 & -0.872143 \\
\hline MCW075_23 & 0.301193 & -0.020668 & 0.069398 & -1.068621 & -0.769590 \\
\hline MCW067_02 & 0.297496 & -0.081443 & 0.058038 & -1.273761 & -0.804913 \\
\hline Slc25a10 & 0.268964 & 0.073774 & 0.045496 & -0.725709 & -0.830849 \\
\hline Usp48 & 0.166792 & 0.011649 & 0.039235 & -0.930159 & -0.764767 \\
\hline Col4al & 0.258305 & 0.043254 & 0.038870 & -0.832545 & -0.849517 \\
\hline$B 2 m$ & 0.127737 & 0.006545 & 0.029585 & -0.948760 & -0.768391 \\
\hline Shcl & 0.257072 & 0.036985 & 0.053275 & -0.856130 & -0.792763 \\
\hline Fstll & 0.177793 & 0.025137 & 0.025407 & -0.858616 & -0.857100 \\
\hline Rgs 2 & 0.174529 & 0.115578 & -0.027143 & -0.337772 & -1.155523 \\
\hline
\end{tabular}

moderate scale of the network. Indeed, setting the threshold a little higher or lower than the average would not disturb the clustered (not paired) relationship of genes considered in this paper.

Step 2. Establishment of connections: Chromosome substitution could affect genes located on the substituted chromosomes and other related chromosomes, which would up-regulate or down-regulate the expression levels of targeted genes, and unrelated genes would keep their expression levels unchanged. Correspondingly, the change ratios of unrelated genes would tend to zero. Therefore, the change ratios could reflect the relationship among genes. We consider the correlations between genes by contrasting the change ratios between any two genes. Specifically, we can compare the change ratios $R_{i}^{\mathrm{I}}$ and $R_{i}^{\mathrm{II}}$ among all 335 nodes. If the trend of changes between two nodes is similar in both $R^{\mathrm{I}}$ and $R^{\mathrm{II}}$ according to (2), then a connection is made between such two nodes (genes):

$$
A_{i j}= \begin{cases}1 & \text { if }\left|R_{i}^{\mathrm{I}}-R_{j}^{\mathrm{I}}\right| \leq T^{\mathrm{I}} \text { and }\left|R_{i}^{\mathrm{II}}-R_{j}^{\mathrm{II}}\right| \leq T^{\mathrm{II}} ; \\ 0 & \text { if }\left|R_{i}^{\mathrm{I}}-R_{j}^{\mathrm{I}}\right|>T^{\mathrm{I}} \text { or }\left|R_{i}^{\mathrm{II}}-R_{j}^{\mathrm{II}}\right|>T^{\mathrm{II}} .\end{cases}
$$

Here, $i, j=1,2, \ldots, 335$ and $i \neq j . A_{i j}=1$ indicates that substitutions of chromosomes 13 and 18 have consistent and similar effects on the genes, while both substitutions could attenuate hypertension; consequently, there is a certain correlation between two genes $i$ and $j$. In such a way, we have constructed the network of hypertension-related genes, which contains 335 nodes (genes) and 1280 edges (connections or links). In Fig. 11 we show the schematic diagram of the gene network for the SS rat with all 335 nodes and an enlarged view of its central part.

\section{Statistical and topological characteristics of gene network}

To analyze the network of hypertension-related genes, we focus on the following indices: degree distributions, sparsity, average path length, clustering coefficient, assortativity and three centrality indices (degree centrality, be- 

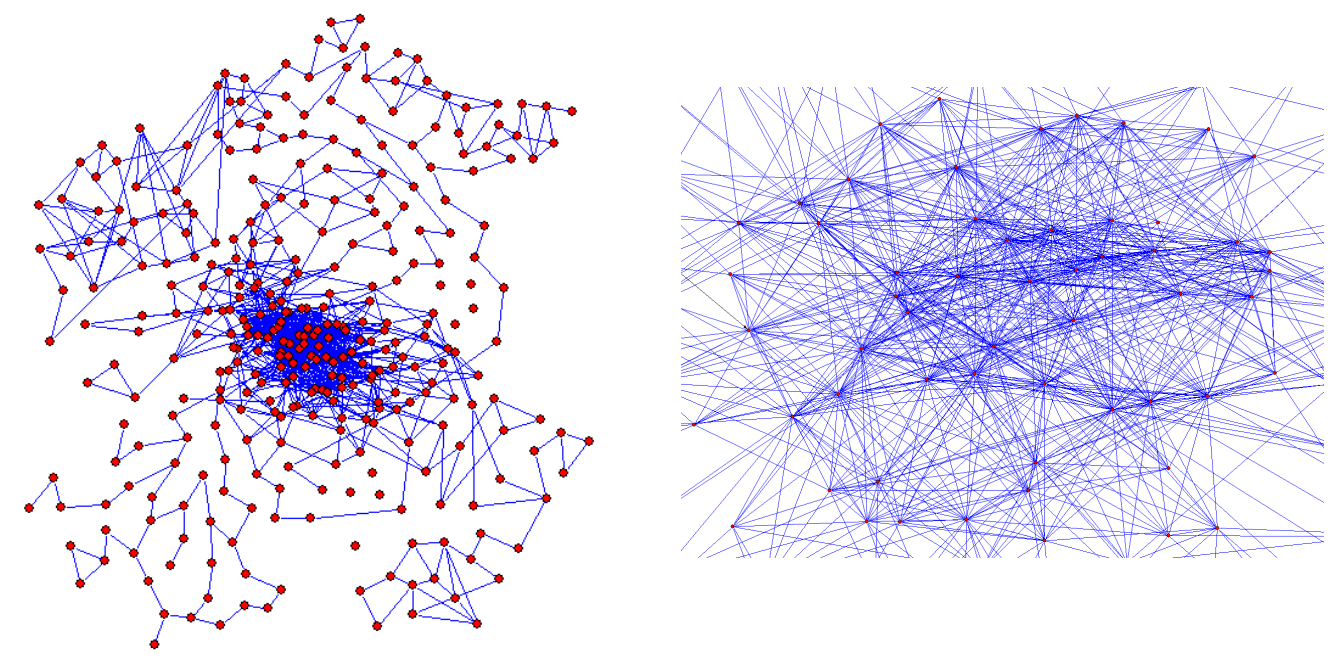

Figure 1: Illustration of the network of hypertension-related genes for the SS rat with all 335 nodes (left) and a magnification of its central part (right).
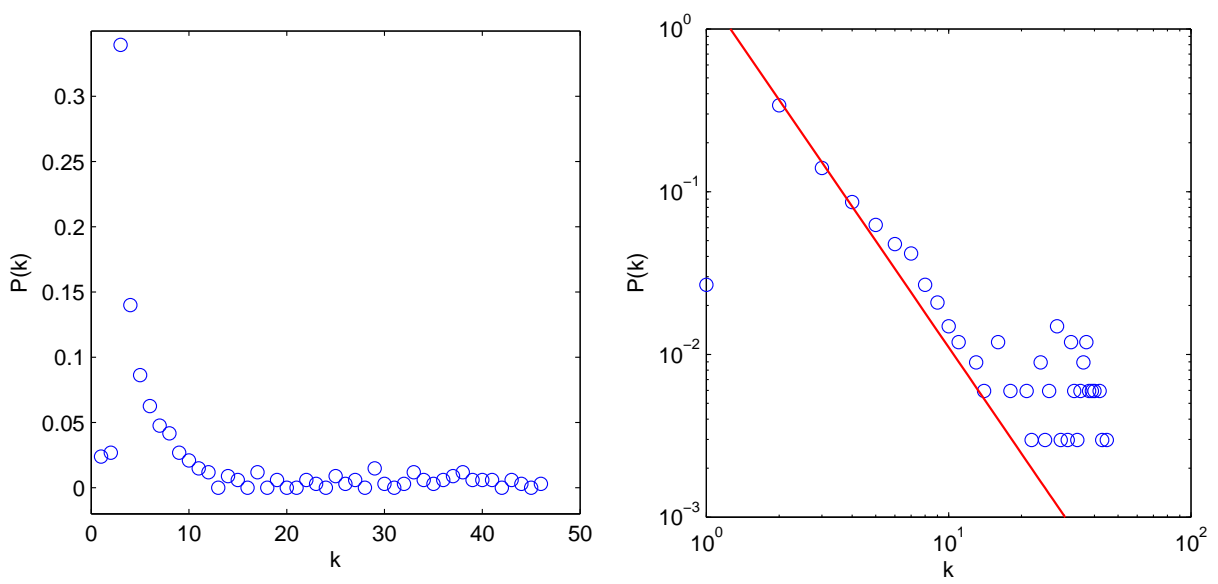

Figure 2: The degree distributions for the network of hypertension-related genes in two coordinates: $k-P(k)$ (left) and $\log$-log degree distribution in logarithmic coordinates (right). In this figure, $P(k)$ denotes the probability of a node having degree $k$. The power-law exponent $\gamma$ is 2.173 .

tweenness centrality and closeness centrality). Further, we will introduce an integrated centrality to determine hub genes in the network.

\subsection{Degree distribution}

The most elementary characteristic of a node is its degree, denoted by $k$, which tells us the number of links (connections or edges) the node has to other nodes. The degree $k_{i}$ of a node $i$ is the total number of its links. The average of $k_{i}$ over all nodes is called the average degree of the network, and is denoted as $\langle k\rangle$. The spread in node degree is characterized by a distribution function $P(k)$, which can quantify the diversity of the whole network. The degree distribution $P(k)$ gives the probability that a randomly selected node has exactly $k$ links (edges). $P(k)$ is obtained by counting the number $N(k)$ of nodes that have $k=1,2, \ldots$ links and dividing by the total number $N$ of nodes [5, 6, 7]. The degree distribution is one of the most important statistical characteristics of networks. 
Fig. 2 plots the degree distributions for the nodes of the gene network in two coordinates. The illustrations show that the probability that a gene can link with $k$ other genes decays as a power-law of the form $P(k) \sim k^{-\gamma}$, where the exponent $\gamma$ is about 2.173 suggesting that the gene network has a scale-free topology. A distinguishing feature of such a scale-free network is the existence of a small number of highly connected nodes, known as hubs, which often determine the network properties and are more important than a large number of other less connected nodes. These hubs correspond to key (feature) genes which play important roles in hypertension.

\subsection{Sparsity}

The gene network involves 335 nodes. If it is fully connected, then the total number of edges of the network is $C_{335}^{2}=55945$. However, the number of edges in our gene network is only 1280, so the ratio between them is $2.288 \%$. From another point of view, the average degree (or average number of connections) $\langle k\rangle$ is about 7.642. Thus it can be seen from both the ratio and the average degree $\langle k\rangle$ that the gene network is sparse.

\subsection{Average path length and clustering coefficient}

We now briefly recall some basic notions of complex networks. Two nodes of a network are connected if a path, namely a sequence of adjacent nodes, links them. There are many alternative paths between two nodes, the path with the smallest number of links (edges) between the selected nodes is called the shortest path. The distance $d_{i j}$ between two nodes $i$ and $j$ is defined as the number of edges along the shortest path connecting them. The diameter $D$ is the maximum distance between any pair of nodes in the network, i.e.

$$
D=\max \left\{d_{i j}\right\}
$$

The average path length (also called characteristic path length) $L$ is defined as the mean distance between two nodes, averaged over all pairs of nodes, i.e.

$$
L=\frac{1}{N(N-1)} \sum_{i \neq j} d_{i j},
$$

here $L$ determines the effective "size" of a network, and offers a measure of the overall navigability of a network [40].

A clustering coefficient can be defined to describe the cohesiveness of the neighborhood of a node [2, 7]. In a network, suppose that a node $i$ has $k_{i}$ edges, the $k_{i}$ nodes are the neighbors of node $i$. The clustering coefficient $c_{i}$ is defined as the ratio between the number $e_{i}$ of edges that actually link the $k_{i}$ neighbors of node $i$ to each other and the total possible number of edges among them, i.e.

$$
c_{i}=\frac{2 e_{i}}{k_{i}\left(k_{i}-1\right)} \quad \text { for } k_{i} \geq 2
$$

The clustering coefficient $C$ of the whole network is the average of $c_{i}$ over all $i$, which characterizes the overall tendency of nodes to form clusters, clearly, $C \leq 1$.

The small-world effect consists of two properties: a short average path length and a relatively high clustering coefficient. In the following, we will give the calculation results about these two properties and show that the gene network is a small-world network.

The gene network exhibits a very short average path length: $L$ is about 5.428, and proportional to the logarithm of the network size $N$, i.e., $L \sim \log (N)$. On the other hand, the maximum distance (i.e., the diameter) $D$ is only 12 . That is, at most twelve hops separate any two genes in the 1280 links of the gene network.

For the gene network, the clustering coefficient is calculated to be $C=0.6063$ according to (5). Compared with the clustering coefficient $C_{\mathrm{E} R}=\langle k\rangle / N=0.0228$ of a corresponding Erdős-Rényi random graph, the clustering coefficient $C$ of the gene network is about 27 times higher than that of the random graph. From what is discussed above, we can draw a conclusion that the gene network has the small-world property characterized by small $L$ and large $C$. 
Table 2: Top 20 values of degree centrality $C_{d}$, betweenness centrality $C_{b}$, closeness centrality $C_{c}$, and integrated centrality $C_{\text {intgr }}$ of hypertensionrelated genes.

\begin{tabular}{|c|c|c|c|c|c|c|c|}
\hline Gene & $C_{d}$ & Gene & $C_{b}$ & Gene & $C_{c}$ & Gene & $C_{\text {intgr }}$ \\
\hline Lcn2 & 0.1347 & Cdk4 & 0.06477 & Col4al & 0.1771 & Col4al & 0.9591 \\
\hline$C t s d$ & 0.1287 & Col4al & 0.06114 & $C d k 4$ & 0.1755 & Lcn2 & 0.9560 \\
\hline Col4al & 0.1257 & Usp48 & 0.05826 & Usp48 & 0.1752 & $C d k 4$ & 0.9525 \\
\hline Fstll & 0.1257 & Lcn 2 & 0.05700 & Nrld 2 & 0.1752 & Fstll & 0.8741 \\
\hline Shcl & 0.1198 & Fstll & 0.04612 & Lcn2 & 0.1750 & Usp48 & 0.8666 \\
\hline$S d c 1$ & 0.1198 & Shcl & 0.04538 & Shcl & 0.1746 & Shcl & 0.8584 \\
\hline$C d k 4$ & 0.1168 & $\operatorname{Nrld} 2$ & 0.04136 & $C d c 2 a$ & 0.1746 & $\operatorname{Nrld} 2$ & 0.8241 \\
\hline MCWA09_96 & 0.1168 & $F z d 2$ & 0.03788 & $F z d 2$ & 0.1743 & $F z d 2$ & 0.8044 \\
\hline $\operatorname{Nrld} 2$ & 0.1138 & $C d c 2 a$ & 0.03555 & Hist1h2ai & 0.1734 & $C d c 2 a$ & 0.7856 \\
\hline$F z d 2$ & 0.1138 & Histlh2ai & 0.03499 & Casp6 & 0.1734 & Histlh2ai & 0.7730 \\
\hline$C d c 2 a$ & 0.1108 & RGICL83 & 0.02879 & Fstll & 0.1730 & Ctsd & 0.7722 \\
\hline Fbnl & 0.1108 & $C t s d$ & 0.02488 & Ctsd & 0.1730 & Casp6 & 0.7191 \\
\hline Timpl & 0.1108 & Casp6 & 0.02453 & Timpl & 0.1730 & Timpl & 0.7183 \\
\hline Hfe & 0.1108 & Timpl & 0.02304 & Ssg1 & 0.1730 & Fbnl & 0.7052 \\
\hline Histlh2ai & 0.1078 & Fbnl & 0.02127 & MCWA09_96 & 0.1712 & MCWA09_96 & 0.7043 \\
\hline Casp6 & 0.1078 & MCW067_02 & 0.02031 & Fbnl & 0.1709 & $S d c 1$ & 0.6925 \\
\hline MCWA13_68 & 0.1078 & MCWA09_96 & 0.01812 & $S d c 1$ & 0.1706 & Ssgl & 0.6263 \\
\hline RGIEJ34 & 0.1048 & RGIHD68 & 0.01539 & MCWA10_48 & 0.1697 & MCWA10_48 & 0.6260 \\
\hline MCWA11_14 & 0.1048 & $S d c 1$ & 0.01460 & $B 2 m$ & 0.1691 & Hfe & 0.6236 \\
\hline$B 2 m$ & 0.1018 & MCW075_23 & 0.01445 & Hfe & 0.1691 & RGIEJ34 & 0.6148 \\
\hline
\end{tabular}

\subsection{Assortativity}

To describe degree correlations between neighboring nodes in a network, the concept of assortativity is introduced [41]. A network is said to be assortative if the nodes with many connections tend to be connected to other nodes with many connections; otherwise, it is said to be disassortative if the nodes with many connections tend to be connected to other nodes with few connections. Most social networks usually exhibit assortativity [10]. The assortativity can be described by measuring the correlation between the degrees of neighboring nodes in terms of the mean Pearson correlation coefficient. For any link $i$, let $x_{i}$ and $y_{i}$ be the degrees of the two vertices connected by the $i$ th edge, with $i=1, \ldots, E$ ( $E$ is the number of edges in the network), then the assortativity coefficient of the network is given by [41]:

$$
r=\frac{E^{-1} \sum_{i} x_{i} y_{i}-\left[E^{-1} \sum_{i} \frac{1}{2}\left(x_{i}+y_{i}\right)\right]^{2}}{E^{-1} \sum_{i} \frac{1}{2}\left(x_{i}^{2}+y_{i}^{2}\right)-\left[E^{-1} \sum_{i} \frac{1}{2}\left(x_{i}+y_{i}\right)\right]^{2}} .
$$

The network is assortative if $r>0$; otherwise it is disassortative if $r<0$.

For the network of hypertension-related genes, the assortativity coefficient is calculated to be $r=0.4065$ from (6). Based on this value, we can say that this gene network exhibits an assortative behavior like most social networks, but unlike most biological networks.

\subsection{Centrality}

\subsubsection{Three centrality indices}

The study of centrality aims at finding out the centralization nodes in the network. There are three centrality indices widely used in network analysis: degree centrality, betweenness centrality, and closeness centrality [42, 43]. These centrality indices determine the relative importance of a node in the network.

The degree centrality of a given node $i$ is the proportion of other nodes that are adjacent to $i$ [43], i.e.

$$
C_{d}(i)=\frac{k_{i}}{N-1},
$$

here $k_{i}$ is the degree of node $i$, and $N-1$ the maximum possible degree of the network. $C_{d}(i)$ is a structural measure of node centrality based on the degree of node $i$.

The second index of node centrality is called betweenness centrality. This index is based upon the frequency with which a node falls between pairs of other nodes on the shortest or geodesic paths connecting them. In a network of 

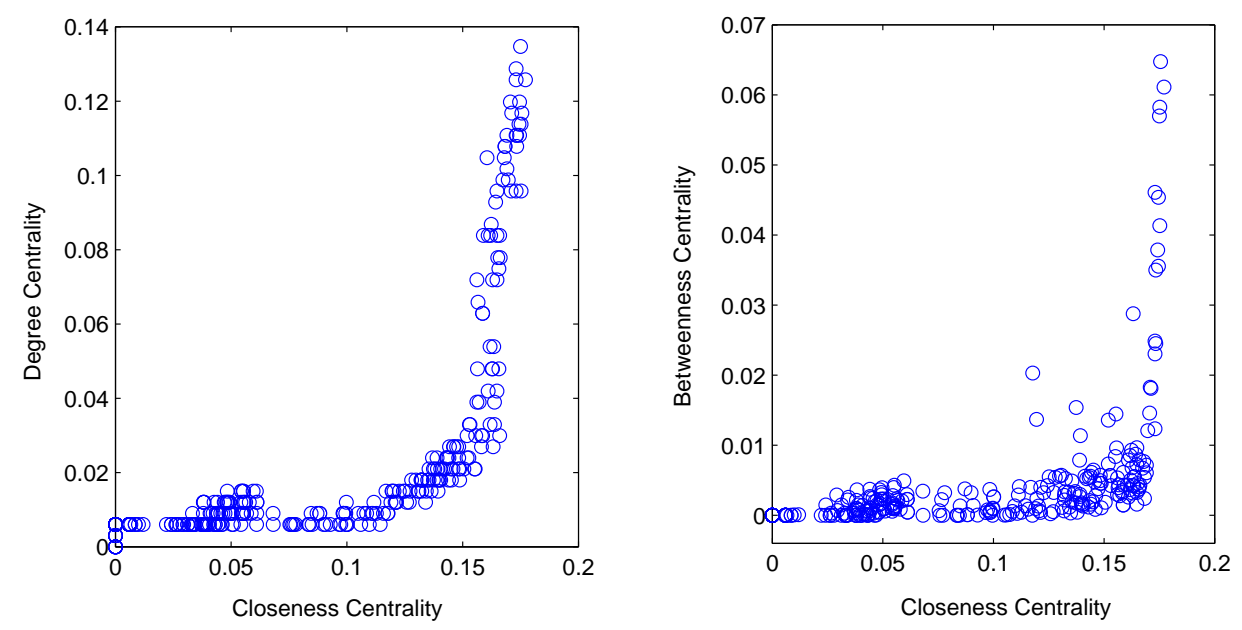

Figure 3: Correspondence among degree centrality $C_{d}$, betweenness centrality $C_{b}$, and closeness centrality $C_{c}$ of hypertension-related genes: $C_{d}$ versus $C_{c}$ (left), and $C_{b}$ versus $C_{c}$ (right).

$N$ nodes, a geodesic is the shortest path between two nodes; the betweenness centrality of a node $i$ is defined as the proportion of all geodesics between pairs of other nodes that include this node $i$ [43]:

$$
C_{b}(i)=\sum_{j(<k)}^{N} \sum_{k}^{N} \frac{g_{j k}(i)}{g_{j k}},
$$

where $g_{j k}$ is the number of geodesics connecting nodes $j$ and $k$, and $g_{j k}(i)$ the number of geodesics connecting the two nodes $j$ and $k$ that contain node $i$.

With the concept of distance described in Section 3.3 , the third index of centrality is defined, which is called closeness centrality. The closeness centrality of a node $i$ is the number of other nodes divided by the sum of the distances between the node $i$ and all others [42, 43]:

$$
C_{c}(i)=\left(L_{i}\right)^{-1}=\frac{N-1}{\sum_{j=1}^{N} d_{i j}},
$$

here, $L_{i}$ is the average distance between the node $i$ and all other nodes, and $d_{i j}$ the distance between nodes $i$ and $j$. For the network of hypertension-related genes, the three centrality indices are calculated and listed in Table2, respectively (we only show the top 20 values of each centrality index).

The degree centrality can be interpreted in terms of the immediate influence of a node in the network, and the betweenness centrality is a measurement of the shortest paths including the node. The closeness centrality depicts how close a node is to all other nodes, and reflects the ability of influence of a node on other nodes through the network. Fig. 3 describes the correspondence among degree centrality $C_{d}$, betweenness centrality $C_{b}$, and closeness centrality $C_{c}$ of hypertension-related genes. The illustration shows that a small number of nodes with high degree and betweenness centrality have almost the same high closeness centrality; while most nodes have low degree and betweenness centrality, whose closeness centrality distributes widely.

\subsubsection{Determination of hub genes}

As calculated above, the network of hypertension-related genes is scale-free, which shows that the network's properties are often determined by a small number of highly connected nodes (i.e., hubs). So the determination of hubs is an important issue. We know that the centrality measurements can accurately and quickly find out relatively important nodes (hubs) in complex networks. In general, one can recognize hubs according to any one of three 


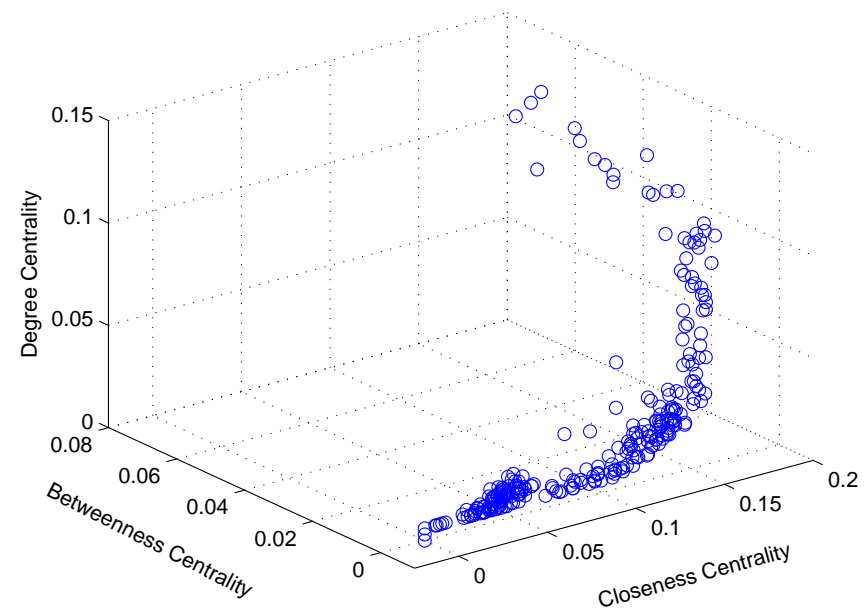

Figure 4: Three-dimensional diagram of degree centrality $C_{d}$, betweenness centrality $C_{b}$, and closeness centrality $C_{c}$ of hypertension-related genes.

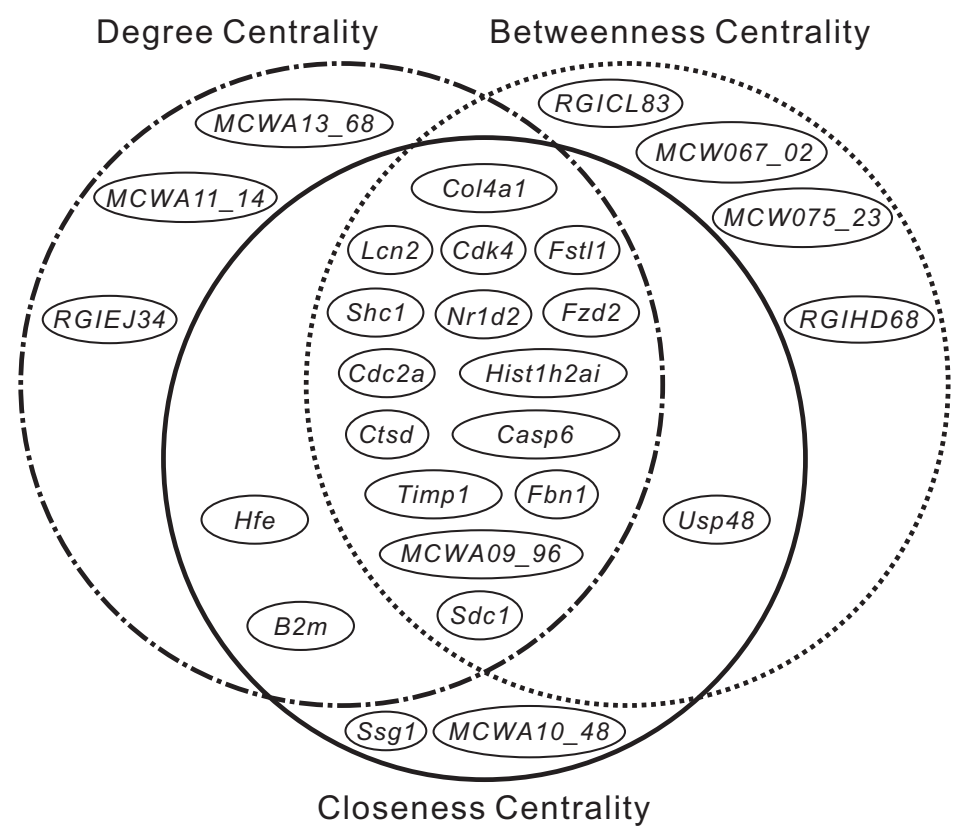

Figure 5: Distribution diagram of some important hypertension-related genes based on analysis of three centrality indices $C_{d}, C_{b}$ and $C_{c}$.

centrality indices $C_{d}, C_{b}$ and $C_{c}$. However, in order to fully reflect the contribution of all these three centrality indices, here we will determine key "hub" genes by considerations from the following two aspects.

(i) Direct consideration from three high centrality indices

We can explore node centrality in the gene network through directly calculating the three centrality indices and analyzing the relationship among them. The betweenness and closeness centrality are based on the shortest path, indicating that local perturbations to hub genes could spread to the whole network very rapidly. Fig. 4illustrates the three-dimensional diagram of degree centrality, betweenness centrality and closeness centrality of the gene network. It can be seen from Fig. 4 that a small number of nodes (hubs) have high values of three centrality indices.

Fig. 5 illustrates the distribution of certain genes according to the values of three centrality indices $\left\{C_{d}, C_{b}, C_{c}\right\}$ in Table 2, where each circle shows the top 20 genes of each centrality index. Thus the intersection of three circles 


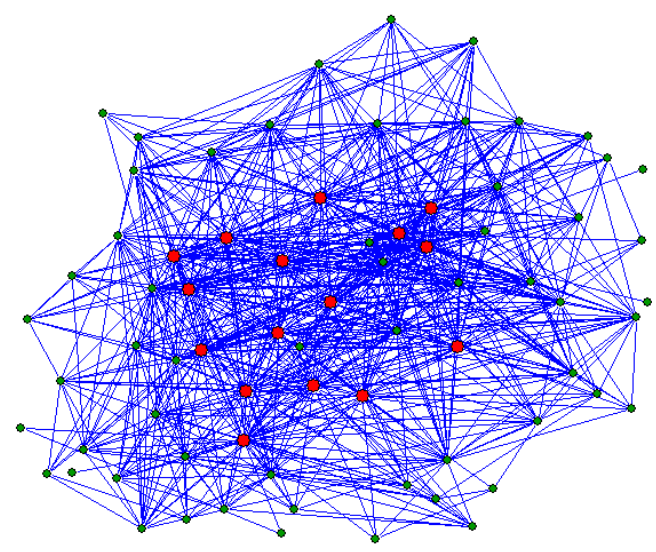

Figure 6: Illustration of all connections of 16 hub genes (big red dots) with other genes (small green dots).

directly gives 15 important hub genes in the network of hypertension-related genes.

(ii) Quantitative consideration from integrated centrality

We can also quantitatively determine hub genes by introducing an integrated centrality index. To this end, we first convert (rescale) three centrality indices into three relative centrality indices: $C_{d}(i) / C_{d, \max }, C_{b}(i) / C_{b, \max }$ and $C_{c}(i) / C_{c, \max }$, where $C_{d, \max }, C_{b, \max }$ and $C_{c, \max }$ are the maximums of $\left\{C_{d}\right\},\left\{C_{b}\right\}$ and $\left\{C_{c}\right\}$, respectively. Then we can introduce an integrated centrality of node $i$, defined as the average of them:

$$
C_{\text {intgr }}(i)=\frac{1}{3}\left[C_{d}(i) / C_{d, \max }+C_{b}(i) / C_{b, \max }+C_{c}(i) / C_{c, \max }\right] .
$$

Obviously, $C_{\mathrm{intgr}}(i)$ has a value between 0 and 1 . For different nodes (genes), the values of $C_{\mathrm{intgr}}$ can be easily calculated, and the top 20 values of $C_{\text {intgr }}$ are listed in Table 2. We observe that the first three genes of the top $3 C_{\text {intgr }}$ values correspond to the maximums of the three centrality indices, respectively: Col4al $\left(C_{c, \max }=0.1771\right)$, Lcn 2 $\left(C_{d, \max }=0.1347\right)$, and $C d k 4\left(C_{b, \max }=0.06477\right)$. We can see that for the top 16 genes of $C_{\text {intgr }}>0.69$, except for the gene Usp48, all other 15 genes are consistent with hub genes determined in (i). Although not included in hub genes in (i) (for the reason that the degree centrality does not enter the top 20 values), $U s p 48$ has a high value of $C_{\text {intgr }}$ due to its high closeness and betweenness centrality, so we should include it in hub genes.

Thus, combining with considerations (i) and (ii), we can determine 16 important hub genes in the network of hypertension-related genes: Col4al, Lcn2, Cdk4, Fstll, Usp48, Shc1, Nrld2, Fzd2, Cdc2a, Histlh2ai, Ctsd, Casp6, Timp1, Fbn1, MCWA09_96, and Sdc1. Fig.6] shows all connections of these 16 hub genes with other genes. These hub genes have the integrated centrality of $C_{\text {intgr }}>0.69$, which is about $70 \%$ of its maximum (0.9591); however, it should be indicated that there is no strict significance threshold for $C_{\mathrm{intgr}}$, and one can also lower the threshold to enable more genes to be included in hub genes.

Moreover, as shown in Figs. 3 and 4, we can observe that most nodes with small degree have widely distributed closeness centrality, indicating that despite of low value of degree, a node could have major impact in the network due to high closeness centrality. Therefore, for the above two considerations, since $C_{\text {intgr }}$ can comprehensively and quantitatively reflect the contribution of three centrality indices $C_{d}, C_{b}$ and $C_{c}$, we suggest to determine hub genes by using $C_{\text {intgr }}$ (i.e., consideration (ii)) for simplicity.

\section{Biological descriptions of hub genes}

In the gene network, hubs typically correspond to key genes and are closely related to hypertension. In Table 3 we list the official full names, gene IDs, and biological functions of 16 hub genes (cf. Refs. [44, 45, 46, 47, 48, 49, 50, $51,52,53,54,55,56,57,58,59,60,61,62,63])$. Also, in the following we choose 8 hub genes to give briefly their biological descriptions according to existing research. 
Table 3: Biological functions of specific hub genes playing important roles in hypertension.

\begin{tabular}{|c|c|c|c|}
\hline Gene & Official full name & Gene ID & Function \\
\hline Col4al & collagen, type IV, alpha 1 & 290905 & Related to epithelial cell differentiation $[44,45]$ \\
\hline Lcn2 & lipocalin 2 & 170496 & Related to cellular response to hydrogen peroxide [46] \\
\hline$C d k 4$ & cyclin-dependent kinase 4 & 94201 & Related to circadian rhythm; organ regeneration [47] \\
\hline Fstll & follistatin-like 1 & 79210 & Related to aflatoxin $\mathrm{B}_{1}$; ammonium chloride [48] \\
\hline Usp48 & ubiquitin specific peptidase 48 & 362636 & Related to ubiquitin-dependent protein catabolic process [49] \\
\hline Shcl & SHC transforming protein 1 & 85385 & $\begin{array}{l}\text { Related to activation of MAPK (mitogen-activated protein kinase) activity } \\
\text { [50] }\end{array}$ \\
\hline $\operatorname{Nrld} 2$ & $\begin{array}{l}\text { nuclear receptor subfamily } 1 \text {, group D, } \\
\text { member } 2\end{array}$ & 259241 & Related to regulation of transcription [51] \\
\hline$F z d 2$ & frizzled family receptor 2 & 64512 & Related to cellular response to growth factor stimulus $[52,53]$ \\
\hline$C d c 2 a(C d k l)$ & cyclin-dependent kinase 1 & 54237 & Related to cellular response to hydrogen peroxide and cell aging [54] \\
\hline Hist1h2ai & histone cluster 1, H2ai & 502129 & Related to nucleosome assembly [55] \\
\hline Ctsd & cathepsin D & 171293 & Related to autophagy; proteolysis; autophagic vacuole assembly $[56,57]$ \\
\hline Casp6 & caspase 6 & 83584 & Related to acute inflammatory response to non-antigenic stimulus [58] \\
\hline Timpl & TIMP metallopeptidase inhibitor 1 & 116510 & Related to aging; cartilage development; cell activation [59] \\
\hline Fbn1 & fibrillin 1 & 83727 & Related to kidney development; heart development $[60,61]$ \\
\hline MCWA09_96 (Ppp3ca) & $\begin{array}{l}\text { protein phosphatase } 3 \text {, catalytic subunit, } \\
\text { alpha isoform }\end{array}$ & 19055 & Related to cardiac hypertrophy [62] \\
\hline$S d c 1$ & syndecan 1 & 25216 & Related to inflammatory response [63] \\
\hline
\end{tabular}

The gene Col4al encodes the major type IV alpha collagen chain of basement membranes. It has been indicated that mutations in Col4al result in a complex vascular phenotype encompassing defects in maintenance of vascular tone, endothelial cell function and blood pressure regulation [45]. Other studies have suggested that a SNP (singlenucleotide polymorphism) in the Col4al gene is strongly associated with PWV (pulse wave velocity), an established independent predictor of adverse cardiovascular outcomes [44].

The gene Lcn2, which has the most connections in the gene network, is a recently identified adipokine that belongs to the superfamily of lipocalins. It is recognized as a biomarker of obesity and inflammation, which are both risk factors for hypertension. Recent findings suggest that genetic variants in Lcn2 may affect blood pressure [46], and adiponectin has multiple protective effects on vascular endothelium.

The gene $C d k 4$ is a positive regulator of the cell cycle, which plays a role in cell cycle progression. Angiotensin II, an essential factor for hypertension, is an important modulator of cell growth through $\mathrm{AT}_{1}$ receptors. Studies have reported when $\mathrm{AT}_{1}$ receptors are stimulated in vivo, DNA synthesis is enhanced in blood vessels by activation of cyclin D1 and $C d k 4$ [47].

The gene Usp48 encodes a protein containing domains that associate it with the peptidase family C19. It is also known as the family 2 of ubiquitin carboxyl-terminal hydrolases, whose family members function as de-ubiquitinating enzymes, recognizing and hydrolyzing the peptide bond at the C-terminal glycine of ubiquitin. Recent data demonstrate that via the inhibition of $U s p 48$, agonist activation of $\mathrm{D}_{3} \mathrm{R}$ (dopamine $\mathrm{D}_{3}$ receptor gene) promotes the degradation of NHE3 (sodium/hydrogen exchanger isoform 3), and thus engenders natriuresis and regulates blood pressure [49].

The gene $F z d 2$ belongs to a class of highly conserved genes, which acts as cell-surface receptors for Wnt proteins. There is evidence that $\mathrm{Wnt} / \mathrm{Fzd}$ signaling is involved in the formation and remodeling of the vasculature. Some existing data also show that $F z d 2$ expressed in aortic smooth muscle cells is modulated by Ang II, and Ang II plays a crucial role in blood pressure regulation and cardiovascular homeostasis, both in vitro and in vivo [52, 53].

The gene $C t s d$ encodes a lysosomal aspartyl protease. This proteinase has a variety of biological functions, such as degradating hemoglobin, serum albumin and myoglobin in endosome. It has been reported that genetic variation of the aspartic proteinases may have an effect on specific clinical diseases such as hypertension and ulcers [56]. Other research suggests that $C t s d$ significantly positively correlates with the arterial hypertension stage as well as with histological grading of atherosclerotic lesions [57].

The gene Timpl is linked to extracellular matrix fibrosis and is elevated in hypertension. Hypertension results in structural changes to the cardiac and vascular extracellular matrix (ECM). Matrix metalloproteinases (MMP), and their inhibitors (TIMP) may play a central role in the modulation of this matrix. Existing observations suggest a possible role for these surrogate markers of tissue ECM composition and the prognosis of cardiovascular events in hypertension [59].

The gene Fbnl encodes a member of the fibrillin family. Recently, it is reported that Fbnl plays an important role in maintaining the physiological arterial stiffness of essential hypertension [60]. Meanwhile, Fbn1 is a major 
component of the microfibrils that form a sheath surrounding the amorphous elastin. Defects in the $F b n 1$ gene are associated with an increased risk of prevalent hypertension. The investigation also reveals that $F b n 1$ may contribute to glomerular damage in hypertensive and diabetic kidney disease [61].

Since our network model is constructed by considering the correlations of GED, hub genes which have similar expression patterns with most of the genes with changed expression levels are typical representatives of the genes with strong correlation. From relevant research literature and the biological functions of these hub genes shown in Table 3 , we know that these hub genes are key (feature) genes that play important roles in hypertension. This shows that our construction of the gene network is suitable.

\section{Concluding remarks}

Hypertension has the complexity and diversity of the genetic factors and pathogenesis. However, a comprehensive understanding of hypertension is still extremely deficient. In this study, based on microarray data, we attempt to reversely obtain the relationships between the hypertension-related genes, rebuild the structure of the gene network by visualization technology, and try to abstract the complex interactions between genes through calculating statistical characteristics of the gene network.

The network of hypertension-related genes is sparse, which has the following characteristics: (i) The gene network shows a scale-free property with power-law degree distribution. A few nodes with large values of three centrality indices $\left\{C_{d}, C_{b}, C_{c}\right\}$ (or equivalently with large values of integrated centrality $C_{\text {intgr }}$ ) correspond to hub genes; they are key (feature) genes involved in the formation of hypertension. (ii) The gene network has a small average path length $(L=5.428)$ and a large clustering coefficient $(C=0.6063)$, i.e., the small-world property, indicating that the local disturbance to hubs would rapidly transfer to the whole network. This property reveals the direct influence of these hub genes on hypertension from another perspective, and implies possible novel molecular genetic signals. (iii) The gene network of hypertension is a description of an abnormal state (illness), which exhibits assortative feature (assortativity coefficient $r=0.4065$ ) unlike most biological networks. The nodes with many connections tend to be connected to those with the similar type, showing that besides being affected by a few feature genes, hypertension is the consequence of accumulation of genetic changes and interaction caused by a variety of factors; this has been demonstrated by the existing research findings. Therefore, it is necessary to focus on further study of the interplay between genes in biological experiments.

In this paper, we construct the network model of hypertension-related genes in genetic level based on both biological knowledge and network theory. The hub genes (Col4a1, Lcn2, Cdk4, etc.) in our network have been confirmed by biological/medical research to play important roles in hypertension. Furthermore, the network can also be analyzed based on actual functional correlation metrics with other threshold selection methods (e.g., [64]) to get more biological information about hypertension in the future; it is believable that the results derived from those methods would be consistent and harmonic in comparison with the theoretical analysis of this paper, and they would complement each other. This study provides another perspective on expounding the molecular genetic mechanism, prevention, and individualization treatment of salt-sensitive hypertension. Meanwhile, the research may hopefully shed light on the development of network-based models (e.g., directed and/or weighted networks) of hypertension and other serious diseases, and explore the mutual regulatory relationships between genes of complex diseases, as well as contribute to finding new drug targets and developing novel ideas. At last, we expect that the complex network approach can provide an effective tool for analyzing the pathogenesis of critical illness.

\section{Acknowledgments}

This work was supported in part by the National Natural Science Foundation of China (NSFC) (Grant Nos. 10565004 and 11365023) and the Specialized Research Fund for the Doctoral Program of Higher Education of China (SRFDP) (Grant No. 20050673001). Our special thanks go to the authors of Ref. [36] for providing the online GED of the SS rat and two consomic rat strains, which are the basis of construction of our network model. The authors would like to thank Prof. S.-L. Peng and Dr. J.-J. Hao for their helpful discussions and suggestions, and the anonymous reviewer for calling our attention to Ref. [64]. 


\section{References}

[1] P. Erdős, A. Rényi, On the evolution of random graphs, Publ. Math. Inst. Hung. Acad. Sci. 5 (A) (1960) $17-61$.

[2] D.J. Watts, S.H. Strogatz, Collective dynamics of 'small-world' networks, Nature 393 (6684) (1998) 440-442.

[3] A.-L. Barabási, R. Albert, Emergence of scaling in random networks, Science 286 (5439) (1999) 509-512.

[4] S.H. Strogatz, Exploring complex networks, Nature 410 (6825) (2001) 268-276.

[5] S.N. Dorogovtsev, J.F.F. Mendes, Evolution of networks, Adv. Phys. 51 (4) (2002) 1079-1187.

[6] R. Albert, A.-L. Barabási, Statistical mechanics of complex networks, Rev. Mod. Phys. 74 (1) (2002) $47-97$.

[7] M.E.J. Newman, The structure and function of complex networks, SIAM Rev. 45 (2) (2003) 167-256.

[8] A. Broder, R. Kumar, F. Maghoul, P. Raghavan, S. Rajagopalan, R. Stata, A. Tomkins, J. Wiener, Graph structure in the Web, Computer Networks 33 (1-6) (2000) 309-320.

[9] A.-L. Barabási, R. Albert, H. Jeong, Scale-free characteristics of random networks: the topology of the world-wide web, Physica A 281 (1-4) (2000) 69-77.

[10] M.E.J. Newman, J. Park, Why social networks are different from other types of networks, Phys. Rev. E 68 (3) (2003) 036122.

[11] J. Camacho, R. Guimerà, L.A.N. Amaral, Robust patterns in food web structure, Phys. Rev. Lett. 88 (22) (2002) 228102.

[12] C. von Ferber, T. Holovatch, Yu. Holovatch, V. Palchykov, Public transport networks: empirical analysis and modeling, Eur. Phys. J. B 68 (2) (2009) 261-275.

[13] W.-X. Wang, C.-Y. Yin, G. Yan, B.-H. Wang, Integrating local static and dynamic information for routing traffic, Phys. Rev. E 74 (1) (2006) 016101.

[14] H. Jeong, B. Tombor, R. Albert, Z.N. Oltvai, A.-L. Barabási, The large-scale organization of metabolic networks, Nature 407 (6804) (2000) $651-654$.

[15] A.-L. Barabási, Z.N. Oltvai, Network biology: understanding the cell's functional organization, Nature Rev. Genet. 5 (2) (2004) 101-113.

[16] C. Böde, I.A. Kovács, M.S. Szalay, R. Palotai, T. Korcsmáros, P. Csermely, Network analysis of protein dynamics, FEBS Lett. 581 (15) (2007) 2776-2782.

[17] L. Diambra, Coarse-grain reconstruction of genetic networks from expression levels, Physica A 390 (11) (2011) $2198-2207$.

[18] F. Karlsson, M. Hörnquist, Order or chaos in Boolean gene networks depends on the mean fraction of canalizing functions, Physica A 384 (2) (2007) 747-757

[19] R. Pastor-Satorras, E. Smith, R.V. Solé, Evolving protein interaction networks through gene duplication, J. Theoret. Biol. 222 (2) (2003) 199-210.

[20] M. Tsuchiya, K. Selvarajoo, V. Piras, M. Tomita, A. Giuliani, Local and global responses in complex gene regulation networks, Physica A 388 (8) (2009) 1738-1746.

[21] E. de Silva, M.P.H. Stumpf, Complex networks and simple models in biology, J. R. Soc. Interface 2 (5) (2005) $419-430$.

[22] M.D. Jankowski, C.S. Henry, L.J. Broadbelt, V. Hatzimanikatis, Group contribution method for thermodynamic analysis of complex metabolic networks, Biophys. J. 95 (3) (2008) 1487-1499.

[23] C.J.L. Murray, A.D. Lopez, Global mortality, disability, and the contribution of risk factors: Global Burden of Disease Study, Lancet 349 (9063) (1997) 1436-1442.

[24] V.L. Burt, J.A. Cutler, M. Higgins, M.J. Horan, D. Labarthe, P. Whelton, C. Brown, E.J. Roccella, Trends in the prevalence, awareness, treatment, and control of hypertension in the adult US population: data from the Health Examination Surveys, 1960 to 1991 , Hypertension 26 (1) (1995) 60-69.

[25] M.H. Weinberger, N.S. Fineberg, S.E. Fineberg, M. Weinberger, Salt sensitivity, pulse pressure, and death in normal and hypertensive humans, Hypertension 37 (2) (2001) 429-432.

[26] M.H. Alderman, Salt, blood pressure and health: a cautionary tale, Int. J. Epidemiol. 31 (2) (2002) 311-315.

[27] B. Rodriguez-Iturbe, N.D. Vaziri, Salt-sensitive hypertension-update on novel findings, Nephrol. Dial. Transplant. 22 (4) (2007) $992-995$.

[28] J.P. Rapp, Genetic analysis of inherited hypertension in the rat, Physiol. Rev. 80 (1) (2000) 135-172.

[29] A.W. Cowley Jr., The genetic dissection of essential hypertension, Nature Rev. Genet. 7 (11) (2006) 829-840.

[30] L.K. Dahl, M. Heine, L. Tassinari, Effects of chronic excess salt ingestion: evidence that genetic factors play an important role in susceptibility to experimental hypertension, J. Exp. Med. 115 (6) (1962) 1173-1190.

[31] J.P. Rapp, Dahl salt-susceptible and salt-resistant rats: a review, Hypertension 4 (6) (1982) 753-763.

[32] A.Y. Deng, In search of hypertension genes in Dahl salt-sensitive rats, J. Hypertens. 16 (12) (1998) $1707-1717$.

[33] A.W. Cowley Jr., R.J. Roman, H.J. Jacob, Application of chromosomal substitution techniques in gene-function discovery, J. Physiol. 554 (1) (2004) 46-55

[34] A.W. Cowley Jr., R.J. Roman, M.L. Kaldunski, P. Dumas, J.G. Dickhout, A.S. Greene, H.J. Jacob, Brown Norway chromosome 13 confers protection from high salt to consomic Dahl S rat, Hypertension 37 (2) (2001) 456-461.

[35] B. Joe, N.E. Letwin, M.R. Garrett, S. Dhindaw, B. Frank, R. Sultana, K. Verratti, J.P. Rapp, N.H. Lee, Transcriptional profiling with a blood pressure QTL interval-specific oligonucleotide array, Physiol. Genomics 23 (3) (2005) 318-326.

[36] M. Liang, N.H. Lee, H. Wang, A.S. Greene, A.E. Kwitek, M.L. Kaldunski, T.V. Luu, B.C. Frank, S. Bugenhagen, H.J. Jacob, A.W. Cowley Jr., Molecular networks in Dahl salt-sensitive hypertension based on transcriptome analysis of a panel of consomic rats, Physiol. Genomics 34 (1) (2008) 54-64. All data of $H, S^{\mathrm{I}}$ and $S^{\mathrm{II}}$ are given in three columns (SS2wk, SS13 2wk, and SS18 $2 w k$ ) in Table S2 (Excel file) of Supplemental Figures and Tables of this reference, which can be readily accessed from the website (http://physiolgenomics.physiology.org/content/34/1/54/suppl/DC1).

[37] R. Cooper, J. Cutler, P. Desvigne-Nickens, S.P. Fortmann, L. Friedman, R. Havlik, G. Hogelin, J. Marler, P. McGovern, G. Morosco, L. Mosca, T. Pearson, J. Stamler, D. Stryer, T. Thom, Trends and disparities in coronary heart disease, stroke, and other cardiovascular diseases in the United States: findings of the National Conference on Cardiovascular Disease Prevention, Circulation 102 (25) (2000) $3137-3147$.

[38] K. Wolf-Maier, R.S. Cooper, H. Kramer, J.R. Banegas, S. Giampaoli, M.R. Joffres, N. Poulter, P. Primatesta, B. Stegmayr, M. Thamm, Hypertension treatment and control in five European countries, Canada, and the United States, Hypertension 43 (1) (2004) $10-17$. 
[39] H. Sanada, J.E. Jones, P.A. Jose, Genetics of salt-sensitive hypertension, Curr. Hypertens. Rep. 13 (1) (2011) $55-66$.

[40] J. Xu, Theory and Application of Graphs, Kluwer Academic Publishers, Dordrecht, Boston, London, 2003.

[41] M.E.J. Newman, Assortative mixing in networks, Phys. Rev. Lett. 89 (20) (2002) 208701.

[42] M.A. Beauchamp, An improved index of centrality, Behav. Sci. 10 (2) (1965) 161-163.

[43] L.C. Freeman, Centrality in social networks: conceptual clarification, Social Networks 1 (3) (1978-1979) 215-239.

[44] K.V. Tarasov, S. Sanna, A. Scuteri, J.B. Strait, M. Orrù, A. Parsa, P.-I. Lin, A. Maschio, S. Lai, M.G. Piras, M. Masala, T. Tanaka, W. Post, J.R. O’Connell, D. Schlessinger, A. Cao, R. Nagaraja, B.D. Mitchell, G.R. Abecasis, A.R. Shuldiner, M. Uda, E.G. Lakatta, S.S. Najjar, COL4A1 is associated with arterial stiffness by genome-wide association scan, Circ. Cardiovasc. Genet. 2 (2) (2009) $151-158$.

[45] T. van Agtmael, M.A. Bailey, U. Schlötzer-Schrehardt, E. Craigie, I.J. Jackson, D.G. Brownstein, I.L. Megson, J.J. Mullins, Col4a1 mutation in mice causes defects in vascular function and low blood pressure associated with reduced red blood cell volume, Hum. Mol. Genet. 19 (6) (2010) 1119-1128.

[46] K.-L. Ong, A.W.K. Tso, S.S. Cherny, P.-C. Sham, T.-H. Lam, K.S.L. Lam, B.M.Y. Cheung, Role of genetic variants in the gene encoding lipocalin-2 in the development of elevated blood pressure, Clin. Exp. Hypertens. 33 (7) (2011) 484-491.

[47] Q.N. Diep, M. El Mabrouk, R.M. Touyz, E.L. Schiffrin, Expression of cell cycle proteins in blood vessels of angiotensin II-infused rats: role of $\mathrm{AT}_{1}$ receptors, Hypertension 37 (2) (2001) 604-608.

[48] D. Li, Y. Wang, N. Xu, Q. Wei, M. Wu, X. Li, P. Zheng, S. Sun, Y. Jin, G. Zhang, R. Liao, P. Zhang, Follistatin-like protein 1 is elevated in systemic autoimmune diseases and correlated with disease activity in patients with rheumatoid arthritis, Arthritis Res. Ther. 13 (1) (2011) R17.

[49] I. Armando, V.A.M. Villar, X. Wang, L. Asico, H. Li, C.S. Escano, R. Felder, P.A. Jose, J.E. Jones, Dopamine D 3 receptor decreases NHE3 expression and function by inhibiting the activity of the de-ubiquitinylating enzyme, USP48, in renal proximal tubule cells, Circulation 124 (21 Suppl.) (2011) A16723 (Abstract).

[50] Y. Noda, S. Yamagishi, T. Matsui, S. Ueda, S.-I. Ueda, Y. Jinnouchi, Y. Hirai, T. Imaizumi, The p66 ${ }^{\text {shc }}$ gene expression in peripheral blood monocytes is increased in patients with coronary artery disease, Clin. Cardiol. 33 (9) (2010) 548-552.

[51] M. Lizier, L. Bomba, A. Minuti, F. Chegdani, J. Capraro, B. Tondelli, R. Mazza, M.L. Callegari, E. Trevisi, F. Rossi, P.A. Marsan, F. Lucchini, The nutrigenomic investigation of C57BL/6N mice fed a short-term high-fat diet highlights early changes in clock genes expression, Genes Nutr. 8 (5) (2013) 465-474.

[52] A.M. Goodwin, P.A. D’Amore, Wnt signaling in the vasculature, Angiogenesis 5 (1-2) (2002) 1-9.

[53] M.E. van Gijn, M.J.A.P. Daemen, J.F.M. Smits, W.M. Blankesteijn, The wnt-frizzled cascade in cardiovascular disease, Cardiovasc. Res. 55 (1) (2002) 16-24.

[54] L. Yu, D.A. Quinn, H.G. Garg, C.A. Hales, Gene expression of cyclin-dependent kinase inhibitors and effect of heparin on their expression in mice with hypoxia-induced pulmonary hypertension, Biochem. Biophys. Res. Commun. 345 (4) (2006) $1565-1572$.

[55] Q. Chen, R. Williams, C.L. Healy, C.D. Wright, S.C. Wu, T.D. O'Connell, An association between gene expression and better survival in female mice following myocardial infarction, J. Mol. Cell. Cardiol. 49 (5) (2010) 801-811.

[56] R.T. Taggart, Genetic variation of human aspartic proteinases, Scand. J. Clin. Lab. Invest. 52 (Suppl. 210) (1992) 111-119.

[57] A.V. Timofeeva, L.E. Goryunova, G.L. Khaspekov, O.P. Ilyinskaya, V.N. Sirotkin, E.R. Andreeva, E.M. Tararak, O.S. Bulkina, V.V. Buza, V.V. Britareva, Yu.A. Karpov, R.Sh. Beabealashvilli, Comparative transcriptome analysis of human aorta atherosclerotic lesions and peripheral blood leukocytes from essential hypertension patients, Kardiologiia 49 (9) (2009) 27-38 (in Russian, with English abstract).

[58] B.T. Berhane, C. Zong, D.A. Liem, A. Huang, S. Le, R.D. Edmondson, R.C. Jones, X. Qiao, J.P. Whitelegge, P. Ping, T.M. Vondriska, Cardiovascular-related proteins identified in human plasma by the HUPO Plasma Proteome Project Pilot Phase, Proteomics 5 (13) (2005) 3520-3530.

[59] M.H. Tayebjee, S. Nadar, A.D. Blann, D.G. Beevers, R.J. MacFadyen, G.Y.H. Lip, Matrix metalloproteinase-9 and tissue inhibitor of metalloproteinase-1 in hypertension and their relationship to cardiovascular risk and treatment: a substudy of the Anglo-Scandinavian Cardiac Outcomes Trial (ASCOT), Am. J. Hypertens. 17 (9) (2004) 764-769.

[60] C. Shen, X. Lu, L. Wang, S. Chen, Y. Li, X. Liu, J. Li, J. Huang, D. Gu, Novel genetic variation in exon 28 of FBN1 gene is associated with essential hypertension, Am. J. Hypertens. 24 (6) (2011) 687-693.

[61] A. Hartner, L. Schaefer, M. Porst, N. Cordasic, A. Gabriel, B. Klanke, D.P. Reinhardt, K.F. Hilgers, Role of fibrillin-1 in hypertensive and diabetic glomerular disease, Am. J. Physiol. Renal Physiol. 290 (6) (2006) F1329-F1336.

[62] I. Drozdov, S. Tsoka, C.A. Ouzounis, A.M. Shah, Genome-wide expression patterns in physiological cardiac hypertrophy, BMC Genomics $11(2010) 557$.

[63] N.G. Frangogiannis, Syndecan-1: a critical mediator in cardiac fibrosis, Hypertension 55 (2) (2010) $233-235$.

[64] F. Censi, A. Giuliani, P. Bartolini, G. Calcagnini, A multiscale graph theoretical approach to gene regulation networks: a case study in atrial fibrillation, IEEE Trans. Biomed. Eng. 58 (10) (2011) 2943-2946. 